\title{
Strategies for prevention of spinal-associated hypotension during Cesarean delivery: Are we paying attention?
}

\author{
Vishal Uppal, MBBS, DA, EDRA, FRCA • Dolores M. McKeen, MD, MSc, FRCPC
}

Received: 4 June 2017/Revised: 8 June 2017/Accepted: 5 July 2017/Published online: 12 July 2017

(C) Canadian Anesthesiologists' Society 2017

Cesarean delivery $(\mathrm{CD})$ is one of the most commonly performed surgical procedures, with operative delivery rates of $32 \%$ in North America. For decades, regional anesthesia (RA), when compared with general anesthesia (GA), has been acknowledged as the safer technique during pregnancy. The risk of death during $\mathrm{CD}$ under $\mathrm{GA}$ is consistently higher than the risk of death attributed to RA during CD. Failed intubation accounts for about two-thirds of GA-associated deaths. ${ }^{1}$ Spinal anesthesia (SA) is currently the preferred anesthesia technique for the majority of CDs, both in North America and Europe. ${ }^{2}$

For over 70 years, clinicians providing obstetrical care have recognized maternal hypotension as the most frequent (up to $85 \%$ incidence) and troublesome complication during SA. Untreated spinal-associated hypotension poses serious risks to the mother, compromises neonatal wellbeing, and results in maternal nausea and vomiting and adverse perinatal outcomes such as fetal acidosis. Despite aggressive measures to improve venous return (preload, left uterine displacement, leg wrapping, etc.) obstetric anesthesiologists have failed to eliminate the problem of SA-associated hypotension during CD. In a 2002 editorial by Macarthur, the author referred to the search for "an infallible technique to prevent spinal-induced hypotension" as the "Holy Grail" of obstetric anesthesia. ${ }^{3}$ Considerable research efforts have since been undertaken in obstetric anesthesia practice to find effective preventive and

V. Uppal, MBBS, DA, EDRA, FRCA - D. M. McKeen, MD, MSc, FRCPC $(\square)$

Department of Anesthesia, Perioperative Medicine and Pain Management, Dalhousie University, Nova Scotia Health Authority and IWK Health Centre, Halifax, NS B3H 2Y9, Canada

e-mail: d.mckeen@dal.ca treatment strategies to deal with this important clinical problem. ${ }^{4}$

In this issue of the Journal, Moore et al. present a clinical trial in which they used an up-down sequential methodology to assess the median seated time to prevent hypotension in $50 \%$ of parturients undergoing elective $\mathrm{CD}$ after SA. ${ }^{5}$ This study is a follow-up to their previous work in which hypotension was prevented. Nevertheless, a significant proportion of parturients $(22 \%)$ required additional supplemental analgesia during the peritoneal closure, indicating a clear disadvantage of the prolonged seated time technique. ${ }^{6}$ A pragmatic criticism of this earlier study was the exclusion of intrathecal fentanyl in their SA technique. Fentanyl, routinely added to spinal bupivacaine for $\mathrm{CD}$, improves intraoperative analgesia, prolongs the duration of block, and reduces the incidence of intraoperative nausea and vomiting.

Thus, in an attempt to overcome this earlier shortcoming, the current study used fentanyl $15 \mu \mathrm{g}$ as an additive to two doses of intrathecal bupivacaine. ${ }^{5}$ With the addition of fentanyl $15 \mu \mathrm{g}$ to hyperbaric bupivacaine 11.25 $\mathrm{mg}$ and morphine $150 \mu \mathrm{g}$, the median seated time to prevent hypotension in $50 \%$ of parturients was a little more than two minutes. In the bupivacaine $15 \mathrm{mg}$ group (with the same dose of fentanyl and morphine), the median seated time to avoid hypotension in $50 \%$ of the population was approximately eight minutes. After assuming the supine position, an additional eight to nine minutes were needed (in both groups) to attain satisfactory surgical conditions (sensory block level of T6) prior to skin incision. Only one of the thirty parturients (in the 11.25 $\mathrm{mg}$ group) experienced pain during the peritoneal closure.

The results of this study add to the authors' prior study and appear to resolve the need for supplemental analgesia. The authors conclude by stating that their findings provide 
a starting point for further clinical study. They do not advocate for clinical adoption and acknowledge that extended seated time requires further comparison with the many effective therapies currently utilized for hypotension before its clinical role can be determined.

We too caution Journal readers to consider several factors before adopting the strategy of extended seated time. First, this study was designed and powered such that hypotension is avoided in only $50 \%$ of parturients. The usefulness of an intervention that provides only a $50 \%$ reduction in the incidence of the clinically relevant problem of spinal-associated hypotension is questionable. The authors acknowledge that further work is required to determine the more clinically useful 90 or $95 \%$ effective seating duration. Readers can also appreciate that this would require an even longer extended seating time, with the possibility that the block may be localized to the sacral area (saddle anesthesia) and the risk that the required block height for adequate surgical anesthesia may never be achieved.

Second, further disadvantages associated with the prolonged seated strategy need to be considered. The extra seated time and additional time to attain surgical block will delay surgical start time, which could reduce operating room efficiency, shorten the duration of effective surgical anesthesia, and further impede any clinical utility of the technique.

Perhaps Journal readers should reflect on an important question: Why are Moore et al. exploring the use of extended seating as a novel approach to the prevention of hypotension? In fact, the authors do not address the current evidence, and they do not provide extensive justification for using this novel approach over simply using vasopressors. It is fair to say that the current study findings suggest that an increased seated time after SA for CD may decrease the efficiency and efficacy of SA. With such an obvious disadvantage, the extended seating technique is unlikely to supersede the current evidence that supports the advantages of prophylactic vasopressor infusion.

What is the current evidence? A robust body of literature now exists comprising numerous well-designed clinical trials, evidence synthesis, ${ }^{7}$ and editorials on strategies to address hypotension during $\mathrm{CD}^{3,4,8,9}$ There is ongoing advocacy for maintenance of preload and avoidance of aortocaval compression. Although colloids have been found to be marginally more effective than crystalloids in this setting, considering the multiple disadvantages of colloids (such as pruritis, cost, and anaphylaxis), crystalloid co-loading is generally preferred. Nevertheless, neither colloid nor crystalloid alone has acceptable efficacy in preventing SA-induced hypotension during $\mathrm{CD} .^{8}$
With this evidence and a better understanding of maternal hemodynamics extrapolated from preeclampsia, the focus has now moved from venous return and maintenance of preload to strategies sustaining the arteriolar tone for maintenance of arterial pressure. ${ }^{9,10}$ Contemporary practice in obstetric anesthesia now advocates vasopressor use as the most important strategy to prevent hypotension during $\mathrm{CD} .{ }^{9}$ Importantly, we have also moved away from ephedrine as the first-line vasopressor. ${ }^{4}$ The evidence is now suggesting that phenylephrine is more effective in maintaining maternal blood pressure and reducing the incidence of nausea/vomiting when compared with ephedrine. ${ }^{7}$ Concerns of uteroplacental perfusion with the use of phenylephrine have been resolved, and in fact, ephedrine is associated with lower fetal $\mathrm{pH}$ and decreased base excess. ${ }^{11}$

Additionally, the literature supports regimens of prophylactic phenylephrine infusions as being more effective than intermittent rescue bolus treatment for reducing the incidence, frequency, and severity of hypotension and the associated maternal symptoms in low-risk healthy parturients. ${ }^{12}$ Caution, however, should be exercised extrapolating these findings to the high-risk population, such as those with pregnancy-induced hypertension or placental insufficiency. The evidence continues to evolve in the obese population, with recent findings similar to those in the non-obese group. The combination of fluid co-loading and a vasopressor regime is a highly effective strategy in dealing with spinal-induced hypotension in the $\mathrm{CD}$ population.

Perhaps Journal readers should reflect on another important question: What are the current knowledge gaps or the justification for this novel approach over the use of vasopressors? While the latter is a well-supported and highly effective strategy, we do acknowledge that patients can still experience episodes of hypotension and bradycardia with the potential for reduced cardiac output, which indicates scope for further improvement. Ongoing programs of vasopressor research continue to address this issue and show that low-dose norepinephrine is an effective alternative to phenylephrine, with the advantage of less depression of maternal heart rate and cardiac output. ${ }^{13}$ Norepinephrine has the potential to be the next "novel" ideal agent for maintaining maternal blood pressure during CD SA. Nevertheless, we await the results of further research. Finally, other novel methods for administering vasopressors are being refined, for example, automated computer-controlled systems look promising and may be the future.

There is now compelling evidence that phenylephrine is superior to ephedrine and that continuous infusion is better than intermittent boluses for the prevention of 
hypotension., ${ }^{72}$ Yet, two recent surveys found that the majority of obstetric anesthesiologists still use ephedrine as the first-choice vasopressor and only $15 \%$ use prophylactic phenylephrine infusions. ${ }^{2,14}$ Has a failure in knowledge translation delayed the implementation of prophylactic vasopressor infusions into care?

It is frequently stated in the knowledge translation literature that it takes an average of 17 years for research evidence to disseminate and impact clinical practice. Nine years ago, Ngan Kee et al. published their landmark randomized-controlled trial comparing phenylephrine with ephedrine for maintaining blood pressure during SA for $\mathrm{CD},{ }^{15}$ and subsequently, there have been numerous reviews and editorials supporting the use of phenylephrine. ${ }^{4,7}$ Does this mean that we will have to wait for another eight years to see evidence-based practice integrated with the obstetric anesthesia care we provide for our patients? The "Holy Grail" has been within our sight for quite some time - are we just not paying attention?

\section{Les stratégies de prévention de l'hypotension secondaire à la rachianesthésiee pendant un accouchement par césarienne : $Y$ portons-nous attention?}

L'accouchement par césarienne est l'une des interventions chirurgicales les plus courantes, les taux d'accouchement opératoires atteignant $32 \%$ en Amérique du Nord. Pendant plusieurs décennies, l'anesthésie régionale (AR) a été reconnue comme la technique plus sécuritaire par rapport à l'anesthésie générale (AG) pendant la grossesse. Le risque de décès pendant un accouchement par césarienne sous AG est considérablement plus élevé que celui attribué à une AR au cours de la même intervention. Les échecs d'intubation sont responsables d'environ deux tiers des décès associés à l'AG. ${ }^{1}$ '̀ l'heure actuelle, la rachianesthésie est la technique d'anesthésie privilégiée pour la majorité des accouchements par césarienne, tant en Amérique du Nord qu'en Europe. ${ }^{2}$

Cela fait plus de 70 ans que les cliniciens prodiguant des soins obstétricaux sont conscients que l'hypotension maternelle constitue la complication la plus fréquente (avec une incidence allant jusqu'à $85 \%$ ) et fâcheuse pendant une rachianesthésie. L'hypotension secondaire à la rachianesthésie, si elle n'est pas traitée, pose des risques graves pour la mère, met en danger le bien-être du nouveau-né, et entraîne des nausées et vomissements maternels ainsi que des conséquences périnatales néfastes telles que l'acidose fœtale. Malgré des mesures vigoureuses destinées à améliorer le retour veineux (précharge, déplacement de l'utérus vers la gauche, compression des jambes à l'aide de bandages, etc.), les anesthésiologistes obstétricaux ne sont pas parvenus à éliminer le problème de l'hypotension secondaire à la rachianesthésie pendant les accouchements par césarienne. Dans un éditorial de 2002 signé par Macarthur, l'auteur faisait référence à la recherche d'une « technique infaillible pour prévenir l'hypotension induite par la rachianesthésie » comme le « Saint Graal » de l'anesthésie obstétricale. ${ }^{3}$ Des efforts de recherche considérables ont depuis été déployés dans la pratique de l'anesthésie obstétricale afin de découvrir des stratégies de prévention et de traitement efficaces qui permettent de prendre en charge cet important problème clinique. $^{4}$

Dans ce numéro du Journal, Moore et coll. présentent une étude clinique dans laquelle les auteurs ont utilisé une méthodologie séquentielle en escalier afin d'évaluer le temps médian en position assise nécessaire à prévenir l'hypotension chez $50 \%$ des parturientes subissant un accouchement par césarienne non urgent après une rachianesthésie. ${ }^{5}$ Cette étude suit les travaux antérieurs des auteurs, dans lesquels ils étaient parvenus à prévenir l'hypotension. Toutefois, une importante proportion des parturientes $(22 \%)$ ont nécessité une analgésie d'appoint supplémentaire pendant la fermeture du péritoine, indiquant un désavantage clair de cette technique de prolongement du temps passé en position assise. ${ }^{6}$ Une critique pragmatique de leur étude plus ancienne était l'exclusion du fentanyl intrathécal de leur technique de rachianesthésie. Le fentanyl, généralement ajouté à la bupivacaïne rachidienne pour les césariennes, améliore l'analgésie peropératoire, prolonge la durée du bloc et réduit l'incidence de nausées et vomissements peropératoires.

Dès lors, dans une tentative de surmonter cette lacune précédemment identifiée, les auteurs ont cette fois-ci utilisé $15 \mu \mathrm{g}$ de fentanyl comme adjuvant à deux doses de bupivacaïne intrathécale. ${ }^{5}$ En ajoutant $15 \mu \mathrm{g}$ de fentanyl à $11,25 \mathrm{mg}$ de bupivacaïne hyperbare et $150 \mu \mathrm{g}$ de morphine, le temps moyen en position assise nécessaire à prévenir l'hypotension chez $50 \%$ des parturientes s'élevait à un peu plus de deux minutes. Dans le groupe ayant reçu $15 \mathrm{mg}$ de bupivacaïne (avec les mêmes doses de fentanyl et de morphine), le temps moyen en position assise nécessaire à prévenir l'hypotension chez $50 \%$ des patientes était d'environ huit minutes. Après avoir pris une position allongée, huit à neuf minutes supplémentaires étaient nécessaires (dans les deux groupes) avant d'atteindre des conditions chirurgicales satisfaisantes (niveau supérieur du bloc sensitif à T6) avant l'incision de la peau. Une seule 
des trente parturientes (dans le groupe $11,25 \mathrm{mg}$ ) a ressenti de la douleur pendant la fermeture du péritoine.

Les résultats de cette étude s'ajoutent à l'étude précédente de ces auteurs et semblent résoudre le besoin d'une analgésie supplémentaire. Les auteurs concluent en déclarant que leurs observations constituent un point de départ pour d'autres études cliniques plus approfondies. Ils ne préconisent pas l'adoption clinique de leur méthode et reconnaissent qu'il faudra comparer cette prolongation du temps passé en position assise aux nombreux traitements efficaces actuellement utilisés pour prévenir l'hypotension avant que le rôle clinique de cette méthode ne puisse être déterminé.

Nous souhaitons nous aussi mettre en garde le lecteur du Journal afin qu'il tienne compte de plusieurs facteurs avant d'adopter cette stratégie de prolongation du temps passé en position assise. En premier lieu, cette étude a été conçue et avait la puissance statistique nécessaire à ce que l'hypotension soit évitée chez $50 \%$ des parturientes seulement. L'utilité d'une intervention qui ne procurerait qu'une réduction de $50 \%$ de l'incidence du problème pertinent d'un point de vue clinique de l'hypotension secondaire à la rachianesthésie est discutable. Les auteurs reconnaissent que des travaux supplémentaires sont nécessaires afin de déterminer la durée efficace de positionnement assis utile d'un point de vue clinique, c'est-à-dire à 90 ou $95 \%$. Le lecteur se rendra rapidement compte que cela nécessiterait une période en position assise encore plus longue, entraînant la possibilité d'un bloc exclusivement localisé à la région sacrée (anesthésie en selle) et le risque que la hauteur requise du bloc pour une anesthésie chirurgicale adéquate ne soit jamais atteinte. Deuxièmement, les autres inconvénients associés à cette stratégie de position assise prolongée doivent être considérés. Le temps supplémentaire en position assise et le temps supplémentaire nécessaire à atteindre le bloc chirurgical retarderont l'heure de début de la chirurgie, réduisant potentiellement l'efficience de la salle d'opération, raccourcissant la durée de l'anesthésie chirurgicale efficace, et restreignant encore davantage toute utilité clinique de cette technique.

J'invite le lecteur à réfléchir à une question importante : pour quelle raison Moore et coll. explorent-ils l'utilisation d'une position assise prolongée en tant qu'approche innovante pour prévenir l'hypotension? En fait, les auteurs n'utilisent pas les données probantes actuelles, et ne justifient pas vraiment l'utilisation de cette approche innovante plutôt que d'avoir simplement recours à des vasopresseurs. Les résultats de cette étude suggèrent en fait qu'un temps prolongé en position assise après une rachianesthésie pour un accouchement par césarienne pourrait réduire l'efficience et l'efficacité mêmes de la rachianesthésie. Avec un désavantage si criant, la technique de prolongation du temps passé en position assise a peu de chance de supplanter les données probantes actuelles appuyant les avantages d'une perfusion prophylactique de vasopresseurs.

Quelles sont les données probantes actuelles? Un important corpus de littérature existe aujourd'hui, lequel comprend de nombreuses études cliniques rigoureuses, synthèses de données probantes ${ }^{7}$ et éditoriaux présentant les diverses stratégies existantes pour prendre en charge l'hypotension pendant une césarienne. ${ }^{3,4,8,9}$ Les données s'accumulent pour promouvoir le maintien de la précharge et éviter la compression aorto-cavale. Bien que les colloïdes soient légèrement plus efficaces que les cristalloïdes dans un tel contexte, étant donné les désavantages multiples des premiers agents (prurit, coût, anaphylaxie), l'hydratation concomitante à l'aide de cristalloïdes est généralement privilégiée. Toutefois, ni les colloïdes, ni les cristalloïdes seuls affichent une efficacité acceptable pour prévenir l'hypotension induite par la rachianesthésie pendant une césarienne. ${ }^{8}$

Grâce à ces données probantes et une meilleure compréhension de l'hémodynamie maternelle extrapolée de la prééclampsie, l'attention s'est aujourd'hui déplacée du retour veineux et du maintien de la précharge vers des stratégies permettant de soutenir le tonus artériolaire afin de maintenir la tension artérielle. ${ }^{9,10} \mathrm{La}$ pratique contemporaine, en anesthésie obstétricale, préconise dorénavant l'utilisation de vasopresseurs en tant que stratégie principale pour prévenir l'hypotension pendant un accouchement par césarienne. ${ }^{9}$ Il est important de noter que l'éphédrine n'est actuellement plus considérée comme le vasopresseur de première intention. ${ }^{4}$ Les données probantes suggèrent désormais que la phényléphrine est plus efficace pour maintenir la tension artérielle maternelle et réduire l'incidence de nausées et vomissements comparativement à l'éphédrine. ${ }^{7}$ Les inquiétudes concernant la perfusion utéro-placentaire liées à l'utilisation de phényléphrine ont été résolues; en fait, l'éphédrine est associée à un $\mathrm{pH}$ fotal plus bas ainsi qu'à une augmentation de l'excès de base. ${ }^{11}$

En outre, selon la littérature, les régimes de perfusion de phényléphrine prophylactique sont plus efficaces qu'un traitement à base de bolus intermittents pour réduire l'incidence, la fréquence et la gravité de l'hypotension et les symptômes maternels associés chez des parturientes en bonne santé à faible risque. ${ }^{12} \mathrm{Il}$ faut toutefois faire preuve de prudence si l'on souhaite extrapoler ces données à la population à risque élevé, comme par exemple les femmes présentant une hypertension induite par la grossesse ou une insuffisance placentaire. Les données probantes évoluent quant à la population obèse, les observations récentes étant similaires à celles s'appliquant aux patientes non obèses. La combinaison d'une charge hydrique concomitante et 
d'un régime de vasopresseurs constitue une stratégie très efficace pour prendre en charge l'hypotension secondaire à la rachianesthésie chez les femmes subissant un accouchement par césarienne.

J'invite le lecteur du Journal à réfléchir à une autre question importante : quelles sont les lacunes actuelles de nos connaissances ou les raisons justifiant cette approche innovante plutôt que l'utilisation de vasopresseurs? Bien que les vasopresseurs soient une stratégie bien tolérée et extrêmement efficace, nous sommes conscients que certaines patientes puissent tout de même souffrir d'épisodes d'hypotension et de bradycardie en raison de la réduction potentielle du débit cardiaque, ce qui indique que des progrès sont toujours possibles dans notre prise en charge. Des programmes de recherche sur les vasopresseurs sont en cours et continuent d'étudier la question; ils démontrent que la norépinéphrine à faible dose constitue une alternative efficace à la phényléphrine, ayant l'avantage de provoquer une dépression moindre de la fréquence cardiaque et du débit cardiaque maternels. ${ }^{13}$ La norépinéphrine pourrait potentiellement être le prochain agent 'innovant' idéal pour le maintien de la tension artérielle maternelle pendant un accouchement par césarienne sous rachianesthésie. Nous attendons toutefois les résultats de ces recherches plus poussées. Enfin, d'autres méthodes innovantes d'administration des vasopresseurs sont en cours d'amélioration. Par exemple, les systèmes informatisés automatisés semblent prometteurs et pourraient constituer une solution future.

Des données probantes convaincantes existent aujourd'hui concernant la supériorité de la phényléphrine par rapport à l'éphédrine et des perfusions continues par rapport aux bolus intermittents pour prévenir l'hypotension. ${ }^{7,12}$ Pourtant, deux sondages récents ont révélé que la majorité des anesthésiologistes obstétricaux utilisaient encore l'éphédrine en tant que vasopresseur de première ligne et que seuls $15 \%$ administraient des perfusions prophylactiques de phényléphrine. ${ }^{2,14} \mathrm{Y}$ a-t-il eu un échec du transfert de connaissances, retardant la mise en œuvre des perfusions prophylactiques de vasopresseurs dans les soins?

On lit souvent, dans la littérature sur le transfert des connaissances, qu'il faut en moyenne 17 ans avant que les données probantes de la recherche ne se diffusent et aient un impact sur la pratique clinique. Voilà neuf ans, Ngan Kee et coll. publiaient leur étude randomisée contrôlée célèbre comparant la phényléphrine à l'éphédrine pour le maintien de la tension artérielle pendant une rachianesthésie pour un accouchement par césarienne ${ }^{15}$; par la suite, de multiples comptes rendus et éditoriaux ont appuyé l'utilisation de la phényléphrine. ${ }^{4,7}$ Est-ce que cela veut dire qu'il faudra attendre huit ans encore avant que cette pratique fondée sur des données probantes ne soit intégrée aux soins d'anesthésie obstétricale que nous offrons à nos patientes? Le « Saint Graal» est à notre portée depuis un certain temps - est-ce possible que nous n'y portions pas attention?

Conflicts of interest None declared.

Editorial responsibility This submission was handled by Dr. Philip M. Jones, Associate Editor, Canadian Journal of Anesthesia.

Other associations Dolores M. McKeen has received payments and travel funding for lectures from Merck Canada Inc. She has conducted a clinical trial that was funded by Merck Canada, with total funding approximately Can\$130, 000. She has acted as a consultant for Merck Canada Inc. Vishal Uppal has conducted a clinical trial that was funded by Recro Pharma, PA, USA. None of these associations directly affect the reporting or content of this editorial.

Funding sources None.

Conflit d'intérêt Aucun.

Responsabilité éditoriale Cet article a été traité par Dr Philip M. Jones, rédacteur adjoint, Journal canadien d'anesthésie.

Autres associations Dolores M. McKeen a reçu des paiements et du financement de voyage pour des conférences de Merck Canada Inc. Elle a réalisé une étude clinique financée par Merck Canada, le financement total atteignant environ $130000 \mathrm{CAD}$. Elle a agi à titre de consultante pour Merck Canada Inc. Vishal Uppal a réalisé une étude clinique financée par Recro Pharma, PA, États-Unis. Aucune de ces associations n'affecte directement la communication ni le contenu de cet éditorial.

Sources de financement Aucune.

\section{References}

1. Hawkins JL, Chang J, Palmer SK, Gibbs CP, Callaghan WM. Anesthesia-related maternal mortality in the United States: 19792002. Obstet Gynecol 2011; 117: 69-74.

2. Staikou C, Paraskeva A, Karmaniolou I, Mani A, Chondrogiannis $K$. Current practice in obstetric anesthesia: a 2012 European survey. Minerva Anestesiol 2014; 80: 347-54.

3. Macarthur A. Solving the problem of spinal-induced hypotension in obstetric anesthesia. Can J Anesth 2002; 49: 536-9.

4. Butwick AJ, Columb MO, Carvalho B. Preventing spinal hypotension during caesarean delivery: what is the latest? $\mathrm{Br} \mathrm{J}$ Anaesth 2015; 114: 183-6.

5. Moore A, el-Mouallem E, el-Bahrawy A, et al. An up-down determination of the required seated duration after intrathecal injection of bupivacaine and fentanyl for the hypotension during cesarean delivery. Can J Anesth 2017; 64: this issue. DOI:10. 1007/s12630-017-0931-z

6. Moore A, Bourrassa-Blanchette S, El Mouallem E, et al. The median effective seated time for hypotension induced by spinal anesthesia at cesarean delivery with two doses of hyperbaric bupivacaine: a randomized up-down sequential allocation study. Can J Anesth 2014; 61: 916-21.

7. Heesen $M$, Kolhr $S$, Rossaint $R$, Straube S. Prophylactic phenylephrine for caesarean section under spinal anaesthesia: 
systematic review and meta-analysis. Anaesthesia 2014; 69: 14365.

8. Loubert C. Fluid and vasopressor management for cesarean delivery under spinal anesthesia: continuing professional development. Can J Anesth 2012; 59: 604-19.

9. Sharwood-Smith G, Drummond GB. Hypotension in obstetric spinal anaesthesia: a lesson from pre-eclampsia. $\mathrm{Br} \mathrm{J}$ Anaesth 2009; 102: 291-4.

10. Dyer RA, Reed AR. Spinal hypotension during elective cesarean delivery: closer to a solution. Anesth Analg 2010; 111: 1093-5.

11. Lin FQ, Qiu MT, Ding $X X, F u S K, L i Q$. Ephedrine versus phenylephrine for the management of hypotension during spinal anesthesia for cesarean section: an updated meta-analysis. CNS Neurosci Ther 2012; 18: 591-7.

12. Siddik-Sayyid SM, Taha SK, Kanazi GE, Aouad MT. A randomized controlled trial of variable rate phenylephrine infusion with rescue phenylephrine boluses versus rescue boluses alone on physician interventions during spinal anesthesia for elective cesarean delivery. Anesth Analg 2014; 118: 611-8.

13. Carvalho B, Dyer RA. Norepinephrine for spinal hypotension during cesarean delivery: another paradigm shift? Anesthesiology 2015; 122: 728-30.

14. Lirk P, Haller I, Wong CA. Management of spinal anaesthesiainduced hypotension for caesarean delivery: a European survey. Eur J Anaesthesiol 2012; 29: 452-3.

15. Ngan Kee WD, Khaw KS, Lau TK, Ng FF, Chui K, $\mathrm{Ng} K L$. Randomised double-blinded comparison of phenylephrine vs ephedrine for maintaining blood pressure during spinal anaesthesia for non-elective caesarean section. Anaesthesia 2008; 63: 1319-26. 